\title{
Necessidades básicas insatisfeitas, desnutrição infantil e risco de doenças na vida
}

\author{
Inappropriate basic health conditions, infantile malnourishment and adult illness risk
}

\section{Alejandro Bernardo Pais ${ }^{1}$, Elena Fernandez de Carrera ${ }^{2}$}

\section{RESUMO}

Objetivo: Analisar o estado nutricional de crianças e adolescentes habitantes de uma região com necessidades básicas inadequadamente atendidas a fim de detectar alterações de nutrição e crescimento que possam se tornar fatores de risco para o desenvolvimento de eventos cardiovasculares na idade adulta.

Métodos: Estudo descritivo do tipo transversal, incluindo 136 crianças e adolescentes, entre cinco e 15 anos de idade, pertencentes a duas regiões da Cidade de Santa Fé, Argentina. Foram coletados dados socioeconômicos (escolaridade, causas de absenteísmo escolar e tipo de alimentação), dados clínicos (pressão arterial), dados antropométricos (peso, altura e índice de massa corpórea) e dados laboratoriais (hemograma e perfil lipídico). Os dados foram analisados por meio do teste do quiquadrado e teste exato de Fisher. Utilizou-se a regressão logística para determinar um modelo preditor de hipertensão arterial.

Resultados: A idade média do grupo estudado foi de $10 \pm 2,6$ anos, sendo $49 \%$ do sexo feminino. Observou-se $22 \%$ da amostra com algum tipo de desnutrição, $11 \%$ com sobrepeso ou obesidade, $20 \%$ com hipertensão arterial e $49 \%$ com alguma alteração no perfil lipídico. Pelo modelo de regressão logística, o sexo feminino $(\mathrm{OR}=3,64$; IC95\% $=1,13-11,73) \mathrm{e}$ a relação $\mathrm{LDL} / \mathrm{HDL}$ alterada $(\mathrm{OR}=2,57$; IC95\% $=1,00-6,62)$ aumentaram o risco de hipertensão arterial.

Conclusões: Alterações nutricionais na infância e adolescência podem aumentar o risco de doenças cardiovasculares já nessas faixas etárias e também na vida adulta, principalmente diante da presença de fatores de risco como alteração no perfil lipídico e obesidade, além do sexo feminino.

Instituição: Universidad Nacional del Litoral, Santa Fé, Argentina ${ }^{1}$ Estudante de Medicina, Escuela de Ciencias Médicas, Universidad Nacional del Litoral, Santa Fe, Argentina

2Diretora do Departamento de Matemática da Faculdad de Bioquímica y Ciencias Biológicas da Universidad Nacional del Litoral, Santa Fé, Argentina

Endereço para correspondência:

Elena Fernandez de Carrera

Departamento de Matemática da Faculdad de Bioquímica y Ciencias Biológica da Universidad Nacional del Litoral

Ciudad Universitaria, Paraje el Pozo, Sin número, Casilla de Correo 242

CEP 3000 - Santa Fe/Argentina

E-mail: ecarrera@fbcb.unl.edu.ar
Palavras-chave: antropometria; desnutrição proteicoenergética; obesidade; hipertensão.

\section{ABSTRACT}

Objective: To analyze the nutritional status of children and adolescents living in a region with inadequate care of basic needs in order to detect nutritional and growth problems that are potential risk factors of cardiovascular events in the adult age.

Methods: Cross sectional descriptive study that enrolled 136 children and adolescents between five and 15 years old, living in two regions of Santa Fé City, Argentine. Data collected included: socioeconomic characteristics (schooling, causes of school absenteeism and type of feeding), clinical data (blood pressure), anthropometric data (weight, height and body mass index) and laboratorial data (hemoglobin, hematocrit and blood lipids). Statistical analysis included chi-square test, Fisher exact test, and logistic regression to determine a predictor model of hypertension.

Results: The studied population presented a mean age of age of $10 \pm 2.6$ years, $49 \%$ were female, $22 \%$ presented some type of malnutrition, $11 \%$ overweight or obesity, $20 \%$ hypertension and $49 \%$ had some altered parameter of the blood lipid profile. According to the logistic regression, female gender $(\mathrm{OR}=3.64$; $95 \% \mathrm{CI}=1.13-11.73)$ as well as LDL/HDL cholesterol ratio $(\mathrm{OR}=2.57 ; 95 \% \mathrm{CI}=1.00-6.62)$ increased the risk of arterial hypertension.

Fonte financiadora: bolsa científica da Universidad Nacional del Litoral, projeto No 4-28 - CAl+D 2006 "Modelos matemáticos y estadísticos avanzados. Su aplicación a sistemas químicos y biológicos" no contexto de um Projeto de Extensão de Interesse social (PEIS), ambos aprovados pela Reitoria da Universidad Nacional del Litoral, em Santa Fé, Argentina.

Recebido em: 27/6/08

Aceito em: 10/11/08 
Conclusions: Nutritional problems in infancy and adolescence increase the risk of cardiovascular disease in these ages and, probably, in the adult life, mainly in the presence of risk factors such as serum lipid profile alteration, obesity, and female gender.

Key-words: anthropometry; protein-energy malnutrition; obesity; hypertension.

\section{Introdução}

$\mathrm{Na}$ Argentina, em alguns grupos populacionais, com frequencia crescente se observa a coexistência de problemas nutricionais que se traduzem tanto pelo déficit como pelo excesso de peso, configurando o processo de "Transição Epidemiológica Nutricional” ${ }^{\prime(1,2)}$.

No extremo dos problemas relacionados ao déficit nutricional, encontra-se a desnutrição e suas variantes, aguda e crônica, sendo a última mais importante em consequência das alterações irreversíveis que acarreta no crescimento e desenvolvimento em longo prazo. Ainda que existam variações populacionais, a prevalência de desnutrição crônica mostra uma tendência decrescente em nível mundial, assim como na América Latina, aonde caiu de 25,6\%, em 1980, para $12,6 \%$ em $2000^{(3)}$.

No outro extremo das desordens nutricionais, encontra-se o excesso de peso, especificamente o sobrepeso e a obesidade, que constitui problema de saúde pública classificado como doença crônica não transmissível ${ }^{(4)}$. Sua frequência atinge de forma significativa a população argentina, sendo, segundo estudo realizado entre 1998 e 2001, o sobrepeso de 20,8\% e a obesidade de $5,4 \%$, semelhante ao observado em outros países latino-americanos ${ }^{(5,6)}$. O parâmetro mais aceito atualmente para avaliar a obesidade em crianças e adolescentes é o índice de massa corpórea (IMC), cuja aplicação tem sido extensa devido à facilidade do seu cálculo( ${ }^{(7)}$. O IMC tem uma boa relação com a gordura corporal medida por meio das pregas cutâneas ${ }^{(4)}$.

O sobrepeso e a obesidade têm um impacto significante na saúde física e psicossocial. O estresse psicológico e a estigmatização social produzidos por esses distúrbios nutricionais são tão graves quanto a sua morbidade. $\mathrm{O}$ excesso de peso se soma, em alguns casos, à dislipidemia e à hipertensão arterial como fatores de risco para o aparecimento de eventos cardiovasculares ${ }^{(8,9)}$. O estudo Bogalusa, efetuado pela Academia Americana de Pediatria em 1999, demonstrou que, em crianças com sobrepeso e obesidade, o colesterol total foi 2,4 a 7,1 vezes mais elevados, o LDL colesterol foi 12,6 vezes mais elevado, sendo o LDL (low density liprotein) colesterol e o IMC os fatores mais fortemente relacionados ao aumento de espessura da camada íntima arterial ${ }^{(10,11)}$. Por isso, a Academia Americana de Pediatria, por meio de seu painel de especialistas (National Cholesterol Education Program, NCEP), recomenda a detecção precoce da hipercolesterolemia na infância ${ }^{(12)}$.

Também para a hipertensão arterial se propõe o controle rotineiro da pressão arterial no consultório pediátrico, visando à prevenção dos eventos cardiovasculares na vida adulta. O objetivo é detectar precocemente a doença que, na Argentina, tem prevalência de $10 \%{ }^{(13-15)}$. A prevenção da hipertensão depende também da alimentação adequada, evitando-se o excesso de sal na comida, e do estímulo à atividade física.

Nesse contexto, o objetivo do presente estudo é analisar o estado nutricional de crianças que habitam regiões desfavorecidas da Argentina, que possuem necessidades básicas não atendidas, de modo a detectar alterações nutricionais e de crescimento que possam se tornar fatores de risco para o aparecimento de eventos cardiovasculares na idade adulta.

\section{Métodos}

Trata-se de um estudo transversal, realizado nos bairros Chalet e San Lorenzo (cidade de Santa Fé, Argentina) localizados em região periférica da cidade, correspondendo a assentamentos populacionais com necessidades básicas não satisfeitas. Ambos sofreram as consequências das inundações, tanto no Rio Salado (em 2002) como devido às chuvas (em 2005), com efeitos devastadores na economia e na saúde.

Nesses bairros, atua uma organização não governamental chamada Movimiento Los Sin Techo (MLST), que visa apoiar o desenvolvimento integral dos marginalizados, procurando resolver problemas estruturais dos mais pobres, como alimentação e assistência médica. O MLST oferece uma refeição diária de segunda a sexta em suas instalações - almoço ou lanche, com supervisão nutricional. O grupo também fornece assistência médica consciente da realidade da nutrição infantil.

Incluíram-se crianças e adolescentes, entre cinco e 15 anos de idade, habitantes dos dois bairros nos quais o MLST concentra suas atividades. Esses bairros se caracterizam por uma grande mobilidade dos habitantes. Foram incluídas crianças que já estavam recebendo assistência nutricional e médica e outras que começavam a recebê-la. 
Os dados foram coletados por um grupo de docentes e alunos da Facultad de Bioquímica y Ciencias Biológicas y a la Escuela de Medicina de la Universidad Nacional del Litoral, que faziam parte de um projeto de extensão de interesse social da referida universidade, com habilitação prévia para percentilação, avaliação nutricional e técnicas de coleta de sangue na cadeira de fisiologia humana. Os dados foram coletados em três planilhas especialmente desenhadas para o estudo.

As variáveis socioeconômicas avaliadas foram: escolaridade, atividade física, horas em frente à televisão, ocupação, tipo de comida e lugar onde recebem a alimentação; frequência de faltas à escola e causas mais frequentes de consultas médicas.

Quanto às variáveis antropométricas e clínicas, o peso foi avaliado em balança tipo báscula, com as crianças em posição ortostática, com os pés ao centro da balança e os braços estendidos ao longo do corpo, cabeça alinhada e voltada para frente. A estatura foi verificada por estadiômetro, com extensão de $2 \mathrm{~m}$ e precisão de $0,5 \mathrm{~cm}$, com o paciente em posição ortostática, pés descalços e unidos, braços ao longo do corpo e posição ereta. A mesma posição foi adotada para a mensuração da prega cutânea tricipital com o uso de adipômetro. Tais técnicas antropométricas seguiram os Guias para avaliação do crescimento da Sociedade Argentina de Pediatria (SAP) ${ }^{(16-18)}$.

Os indicadores antropométricos para as avaliações coletivas foram obtidos das Tabelas de Lejarraga, de 1987, publicadas pela SAP ${ }^{(16)}$. Classificaram-se como desnutridos agudos aqueles com relação estatura/peso inferior a $80 \%$ do peso médio correspondente a crianças daquela estatura ${ }^{(17,18)}$. A definição de desnutrição crônica baseou-se na relação estatura/idade inferior ao percentil (P) $3^{(17,18)}$. O IMC foi aplicado para catalogar sobrepeso (IMC entre P85 e P95) e obesidade (acima do P95) ${ }^{(19-21)}$. Quanto à prega cutânea tricipital, utilizou-se como valor de corte o $\mathrm{P} 75^{(17,18)}$.

Para as medidas da pressão arterial, foram adotadas as recomendações da American Heart Association ${ }^{(22)}$ por meio do método indireto com ausculta do pulso. Os manguitos foram selecionados de maneira a ter a largura apropriada, fazendo-se três medidas de pressão, com intervalo de um minuto entre elas. $\mathrm{O}$ valor das três medidas de pressão arterial sistólica e diastólica foi interpretado de acordo com a tabela de percentis recomendada pela Academia Americana de Pediatria ${ }^{(23)}$, que classifica os níveis de pressão em: normal (<P90); pré-hipertensão (igual ao P90 e < P95); hipertensão estádio 1 (entre P95 e P99 mais $5 \mathrm{mmHg}$ ); hipertensão estádio 2 (acima de $5 \mathrm{mmHg}$ do P99).
Quanto às determinações laboratoriais, foi realizada a coleta de sangue após jejum de 12 horas para avaliação de hemograma completo (Cell-dyn 2000), perfil lipídico com colesterol total (CT), triglicérides (TG) e fração HDLColesterol (HDL-C) por método enzimático colorimétrico. A fração LDL-Colesterol (LDL-C) foi calculada pela fórmula de Friedewald ${ }^{(24)}$ e a relação LDL-C/HDL-C foi obtida pela divisão dos valores de LDL-C e HLD-C. O CT, o LDL-C e os TG foram considerados alterados se os valores eram superiores a, respectivamente, $170 \mathrm{mg} / \mathrm{dL}, 110 \mathrm{mg} / \mathrm{dL}$ e $100 \mathrm{mg} / \mathrm{dL}^{(14)}$.

Quanto à anemia, consideraram-se alterados valores de hemoglobina inferiores a $11 \mathrm{~g} / \mathrm{dL}$ para todas as crianças, assim como meninas com idade inferior a dez anos e hemoglobina menor do que $11 \mathrm{~g} / \mathrm{dL}$, meninas entre dez e 12 anos e hemoglobina inferior a $11,5 \mathrm{~g} / \mathrm{dL}$ e meninas com idade superior a 12 anos e valores de hemoglobina inferiores a $12 \mathrm{~g} / \mathrm{dL}$.

Para evitar a influência da idade na análise estatística, optou-se por trabalhar com percentis de peso e estatura. As proporções foram comparadas pelo teste do qui-quadrado ou, quando adequado, pelo teste exato de Fisher. Utilizou-se a regressão logística para modelar um sistema preditor da presença/ausência de hipertensão arterial. As variáveis preditoras (independentes) analisadas foram: LDL-C, prega tricipital e sexo, que permaneciam no modelo apenas se melhorassem o seu ajuste. Aplicou-se o qui-quadrado de Wald para testar a significância do ajuste ${ }^{(25)}$. O teste de Hosmer-Lemeshow indicou a exatidão do modelo de preditor, sendo uma prova da qualidade do ajuste do modelo como um todo ${ }^{(26)}$. Ainda, para medir a capacidade de previsão do modelo construído, construiu-se a curva ROC (Receiver Operating Characteristics Curve), calculando-se a área sob a curva ${ }^{(27)}$. Estimaram-se os coeficientes $\beta$ e o risco relativo e $\beta$, construindo-se os intervalos de confiança de $95 \%{ }^{(27)}$. Todos os testes de hipóteses usaram $\alpha=0,05$. O processamento dos dados foi feito nos softwares SPSS 10.0 e Epi-Info 2000.

\section{Resultados}

A população do estudo foi de 136 crianças e adolescentes (49\% do sexo feminino), com idade média de 10,0 $\pm 2,6$ anos e variação de cinco a 15 anos.

Em termos socioeconômicos e culturais, observou-se que $90 \%$ da população frequentava a escola, sendo que, na faixa etária escolhida, a escola é obrigatória. Dentre as crianças, $66 \%$ recebiam almoço ou merenda no refeitório escolar, alimentação fornecida pela organização não governamental (MLST). Repetiram o ano letivo 18\% dos 
analisados, taxa similar à publicada em 2005 pela Prensa Institucional da Universidade Nacional do Litoral para a cidade de Santa Fé, na Argentina (12 a 15\%) ${ }^{(28)}$. Por outro lado, $71 \%$ das crianças compareciam de modo irregular à escola, sendo a causa mais frequente das faltas o relato de doenças (65\% por parasitose e infecções respiratórias). Do total de crianças, $85 \%$ passavam mais de três horas na frente da televisão e $25 \%$ delas não realizavam atividades físicas. Metade das mães estava desempregada, assim como $10 \%$ dos homens.

Quanto aos dados antropométricos e clínicos, 50\% dos pesos das crianças foram inferiores ao percentil 35 da tabela peso versus idade da Sociedade Argentina de Pediatria (Figura 1). Do grupo analisado, $14,7 \%$ eram desnutridos agudos, 7,5\% desnutridos crônicos, 11,7\% tinham sobrepeso e 2,9\%, obesidade. A comparação desses valores aos de prevalência nacional argentina de desnutrição crônica (12\%) não mostrou diferença significante. Já em relação aos desnutridos agudos, os dados nacionais mostram frequência máxima de 3\% em

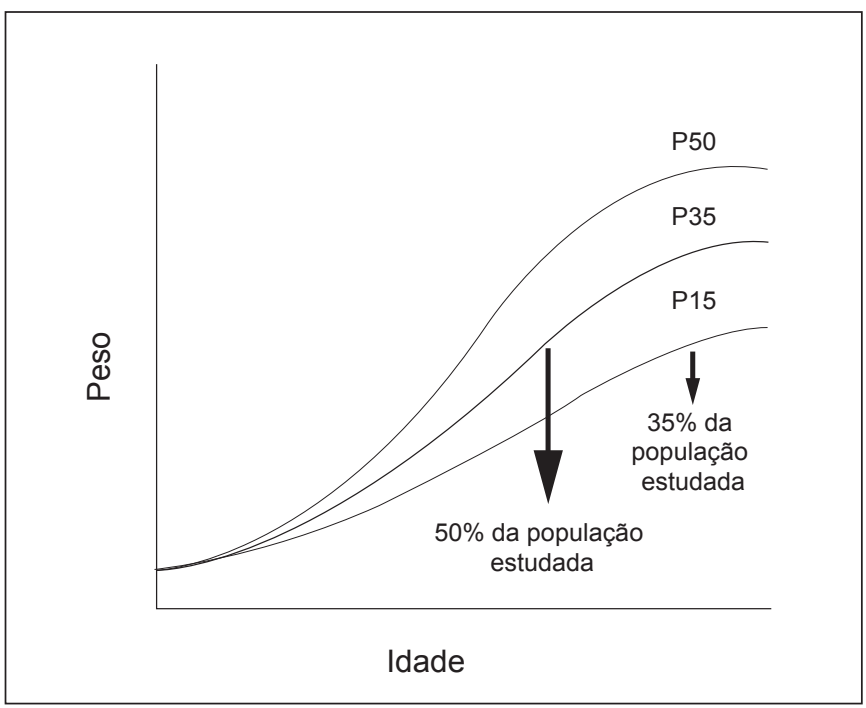

Figura 1 - Porcentual da população estudada segundo os percentis do peso.

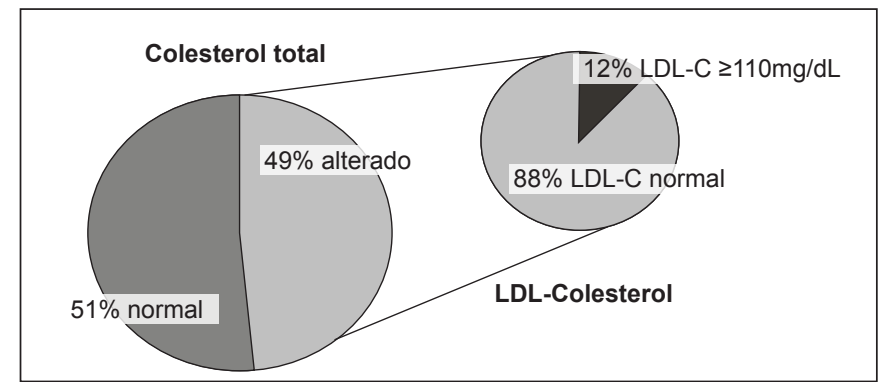

Figura 2 - Porcentual de crianças e adolescentes com perfil lipídico alterado e sua relação com LDL-C alterado. comparação à taxa de $14 \%$ detectada nesse estudo $(p=0,002)$, sendo o poder do teste de pelo menos $85 \%{ }^{(3,29)}$.

O sobrepeso e a obesidade ocorreram mais em mulheres $(n=11)$ do que em homens $(n=4)$, com diferença significativa $(p=0,015)$. Observou-se, também, prega tricipital acima do percentil 75 em sete pacientes.

Quanto à pressão arterial, do total de crianças e adolescentes analisados, $20 \%$ apresentaram valores sugestivos de hipertensão, sendo 17 homens e 9 mulheres $(p=0,025)$. Dos 26 hipertensos, quatro eram obesos e cinco desnutridos. Comparou-se a frequência de crianças com sobrepeso e obesidade portadoras de hipertensão (4/20) com a de desnutridos com pressão arterial acima do percentil 95 (5/30), que se mostrou similar $(p=0,99)$.

Em relação aos exames laboratoriais, a análise do perfil lipídico mostrou alteração em $49 \%$. Do total, $22 \%$ tinham valores de CT alterado no sangue, $12 \%$ tinham o LDL-C elevado e 15\%, os TG alterados (Figura 2). Comparou-se, então, a proporção de crianças e adolescentes com CT e LDL-C elevados entre aqueles que apresentavam ou não hipertensão arterial através do teste de qui-quadrado, observando-se que o fato de a pessoa estudada ser dislipidêmica aumentava em 2,6 vezes a chance de hipertensão arterial (Odds Ratio: 2,63; intervalo de confiança, IC 95\%: 1,07-6,46). Das crianças com hipertensão arterial, nove apresentaram também colesterolemia superior aos valores normais. Nenhum paciente avaliado mostrou-se anêmico.

$\mathrm{Na}$ regressão logística, a variável independente foi a presença de hipertensão arterial nas crianças e adolescentes analisados, de maneira a criar um modelo preditor da doença segundo certas variáveis de interesse. As variáveis independentes testadas foram: sexo, LDL-C/HDL-C, prega tricipital, IMC, triglicérides e estatura. Com a eliminação progressiva dos não significantes, obteve-se o seguinte modelo final:

$\mathrm{y}_{\text {ajustado }}=\frac{e^{z}}{1+e^{z}}$ onde $\mathrm{z}=-2,291+0,945 \mathrm{LDL}-\mathrm{HDL}+1,292$ sexo.

Como o sexo é uma variável dicotômica, o valor e $\beta$ é interpretado como um quociente da Odds Ratio que, por sua vez, estima o risco relativo (RR). Na Tabela 1, observa-se que o risco de hipertensão foi 3,6 vezes maior para o sexo feminino e aumenta 2,5 vezes para cada unidade de aumento do LDL-C/HDL-C. O modelo proposto tem um ajuste adequado (Hosmer-Lemeshow =0,917), com uma média de classificação correta de $78 \%$, apresentando um poder preditor 
satisfatório, uma vez que a área sob a curva ROC construída a partir dos valores de sensibilidade e especificidade foi 0,725 $(p<0,05$, Figura 3).

\section{Discussão}

Da população analisada, $85 \%$ permanece mais de três horas em frente à televisão e $25 \%$ não faz atividade física regular. Tais fatos, em associação a fatores de risco cardiovasculares, aumentam a chance do aparecimento de doenças cardiovasculares na vida adulta ${ }^{(30,31)}$.

Quanto aos desnutridos, dois fatos relevantes merecem discussão. Por um lado, a porcentagem de baixa estatura é menor do que a esperada para a população alvo do estudo e apenas duas crianças com baixa estatura apresentaram também sobrepeso e obesidade. Embora esse achado não seja condizente com o processo de transição epidemiológica nutricional observado nos povos latino-americanos, talvez esteja relacionado ao trabalho da organização não governamental que evita a progressão para fase crônica da desnutriçãa ${ }^{(1,2)}$.

A prevalência de desnutrição aguda, quase seis vezes maior nos dois bairros estudados do que a da população argentina, pode ser explicada pela mobilidade do grupo analisado e pelo pouco tempo que as crianças estiveram sob a tutela da organização não governamental. Existe uma forte migração no país, do interior à metrópole, e ao chegar à metrópole, as crianças têm alta proporção de desnutrição. Além disso, moram em bairros com necessidades básicas inadequadamente atendidas, como os dois bairros estudados neste trabalho.

Os resultados quanto à pressão arterial permitem inferir uma prevalência de hipertensão de $20 \%$, sendo $10 \%$ maior do que revelam os estudos realizados na Argentina. Além disso, a alteração do perfil lipídico aumenta a chance de hipertensão $\operatorname{arterial}^{(13-15)}$.

É notável a ausência da anemia nas crianças e adolescentes incluídos na pesquisa, embora estudos argentinos revelem maior prevalência de anemia justamente na região de proveniência desses pacientes ${ }^{(29)}$. Quando vêm a esses bairros, as crianças recebem da organização não governamental alimentos que obedecem de forma adequada às exigências nutricionais, com oferta de ferro que poderia contribuir para a menor prevalência de anemia aqui constatada.

Pode-se concluir que os problemas nutricionais na infância não trazem como consequência somente a desnutrição e a obesidade, mas também aumentam o risco de transtornos
Tabela 1 - Modelo de regressão logística para a variável resposta "hipertensão arterial"

\begin{tabular}{lccc}
\hline Covariáveis & $\begin{array}{c}\text { B Estimado } \\
\text { ( } \mathbf{p} \text { Wald })\end{array}$ & $\mathbf{e} \boldsymbol{\beta}$ & IC $\mathbf{9 5 \%}$ \\
\hline Constante & $-2,29$ & 0,10 & \\
LDL-C/HDL-C & $(6,36)$ & & \\
& 0,94 & 2,57 & $1,00-6,62$ \\
Sexo & $(3,84)$ & & \\
& 1,29 & 3,64 & $1,13-11,73$ \\
\hline
\end{tabular}

LDL-C: low density lipoprotein colesterol; HDL-C: high density lipoprotein colesterol

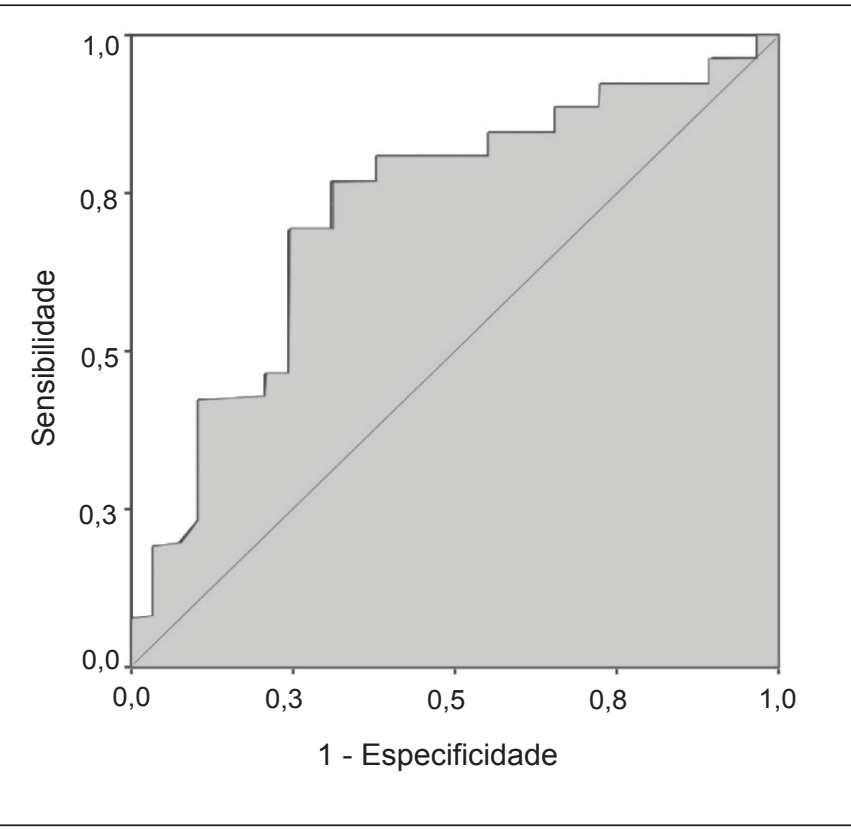

Figura 3 - Curva ROC (Receiver Operating Characteristics Curve) para o modelo hipertensão arterial (o gráfico associado à regressão logística, com área sob a curva de 0,725 , demonstra o poder preditor satisfatório do modelo proposto).

cardiovasculares futuros. Vale ressaltar que a hipertensão arterial esteve associada ao excesso de peso e à desnutrição, sendo seu risco significativamente aumentado nas mulheres. Tudo isso indica a importância do controle nutricional na infância.

\section{Agradecimentos}

Agradecemos a Liliana Ester Contini, Mauro Alexis, Vaira Colombini e Stella Maris, todos docentes do Departamento de Matemática da Universidad Nacional del Litoral. Agradecemos também aos integrantes do Projeto de Extensão de Interesse Social (PEIS): Evolución de los problemas nutricionales en niños de barrios con necesidades básicas 
insatisfechas, dirigido pela professora bioquímica Mahieu Stella Teresita. Ao pessoal encarregado dos efectores Chalet y San Lorenz, pertencentes ao Movimiento Los Sin Techo da cidade de Santa Fé. À Doutora Marta Wagener por sua generosa colaboracão para a solucão de problemas metodológicos que surgiram no desenvolvimento deste estudo e ao Doutor Joel Alvarez Lamounier, que colaborou para a publicação deste artigo.

\section{Referências bibliográficas}

1. Durán P. Transición epidemiológica nutricional o el "efecto mariposa". Arch Argent Pediatr 2005;103:195-7.

2. Orden AB, Torres MF, Luis MA, Cesani MF, Quintero FA, Oyhenart EE. Evaluación del estado nutricional en escolares de bajos recursos socioeconómicos en el contexto de la transición nutricional. Arch Argent Pediatr 2005;103: 205-11.

3. de Onis M, Frongillo EA, Blössner M. Is malnutrition declining? An analysis of changes in levels of child malnutrition since 1980. Bull World Health Organ 2000;78:1222-33.

4. Subcomisión de Epidemiología y Comité de Nutrición. Consenso sobre factores de riesgo de enfermedad cardiovascular en pediatría. Obesidad. Arch Argen Pediatr 2005;103:262:81.

5. Kovalskys I, Bay L, Herscovici CR, Berner E. Prevalencia de obesidad en la población de 10 a 19 años en la consulta pediátrica. Arch Argent Pediatr 2003;101:441-7.

6. Lobstein T, Baur L, Uauy R, IASO International Obesity Task Force. Obesity in children and young children: a crisis in public health. Obes Rev 2004; 5 (Suppl 1):4-104.

7. Barlow SE, Dietz WH. Obesity evaluation and treatment: Expert Committee recommendations. The Maternal and Child Health Bureau, Health Resources and Services Administration and the Department of Health and Human Services. Pediatrics 1998;102:E29.

8. Dirisamer A, Widhalm K. Lipoprotein(a) as a potent risk indicator for early cardiovascular disease. Acta Paediatr 2002;91:1313-7.

9. Wiegman A, Rodenburg J, de Jongh S, Defesche JC, Bakker HD, Kastelein JJ et al. Family history and cardiovascular risk in familial hypercholesterolemia: data in more than 1000 children. Circulation 2003;107:1473-8.

10. Freedman DS, Dietz WH, Srinivasan SR, Berenson GS. The relation of overweight to cardiovascular risk factors among children and adolescents: the Bogalusa Heart Study. Pediatrics 1999;103:1175-82.

11. Li S, Chen W, Srinivasan SR, Bond MG, Tang R, Urbina EM et al. Childhood cardiovascular risk factors and carotid vascular changes in adulthood: the Bogalusa Heart Study. JAMA 2003;290:2271-6.

12. American Academy of Pediatrics, Committee on Nutrition. Cholesterol in childhood. Pediatrics 1998;101:141-7.

13. Grupo de Hipertensión. Consenso sobre factores de riesgo de enfermedad cardiovascular en pediatría. Hipertensión arterial en el niño y el adolescente. Arch Argent Pediatr 2005;103:348-57.

14. Souza MSF, Leme RB, Franco RR, Romaldini CC, Tumas R, Cardoso AL et al. Síndrome metabólica em adolescentes com sobrepeso e obesidade. Rev Paul Pediatr 2007;25:214-20.

15. Dei-Cas SA, Dei-Cas IJ, Dei-Cas P, Szyrma ME, Ciancia L, Comas JP et al. Estudio de la presión arterial en adolescentes de 15 años. Su relación con características antropométricas y factores de riesgo de hipertensión arterial. Arch Argent Pediatr 2000;98:161-70.
16. Sociedad Argentina de Pediatra, Comité Nacional de Crecimiento y Desarrollo. Guías para la evaluación del crecimiento. 2 ed. Buenos Aires: SAP; 2001.

17. Rinaldi AEM, Pereira AF, Macedo CS, Mota JF, Burini RC. Contribuições das práticas alimentares e inatividade física para o excesso de peso infantil. Rev Paul Pediatr 2008;26:271-7.

18. Lejarraga H, Anigstein C, Di Candida A, Henrich J, Krupitzky S, Machado O et al. Criterios de diagnóstico y tratamiento: crecimiento y desarrollo. Buenos Aires: SAP; 1983.

19. Dietz WH, Robinson TN. Use of the body mass index (BMI) as a measure of overweight in children and adolescents. J Pediatr 1998;132:191-3.

20. World Health Organization. Child growth standards. Training course on child growth assessment. Geneva: WHO; 2006.

21. de Onis M, Garza C, Victora CG, Onyango AW, Frongillo EA, Martines J. The WHO Multicentre Growth Reference Study: planning, study design, and methodology. Food Nutr Bull 2004;25 (Suppl 1):S15-26.

22. Perloff D, Grim C, Flack J, Frohlich ED, Hill M, McDonald M et al. Human blood pressure determination by sphygmomanometry. Circulation 1993;88: 2460-70.

23. National High Blood Pressure Education Program Working Group on High Blood Pressure in Children and Adolescents. The fourth report on the diagnosis, evaluation and treatment of high blood pressure in children and adolescents. Pediatrics 2004 (Suppl 2);114:555-76.

24. Friedewald WT, Levy RI, Fredrickson DS. Estimation of the concentration of low-density lipoprotein cholesterol in plasma, without use of the preparative ultracentrifuge. Clin Chem 1972;18:499-502.

25. Bagley SC, White H, Golomb BA. Logistic regression in the medical literature: standards for use and reporting, with particular attention to one medical domain. $\mathrm{J}$ Clin Epidemiol 2001;54:979-85.

26. Altman D. Practical Statistics for Medical Research. $7^{\text {th }}$ ed. London: Chapman \& Hall; 1997.

27. Zweig MH, Campbell G. Receiver-operating characteristic (ROC) plots: a fundamental evaluation tool in clinical medicine. Clin Chem 1993;39:561-77.

28. Santafeciudad.com [homepage on the Internet]. Educación: Repitencia escolar en el sistema educativo santafesino [cited 2008 Mar 28]. Avalable from: http:// santafeciudad.com/modules/news/article.php?storyid=7349

29. Argentina. Ministerio de Salud. Presidencia de la Nación. Encuesta Nacional de Nutrición y Salud [homepage on the Internet]. Documento de Resultados 2007 [cited 2008, Mai 20]. Available from: http://www.msal.gov.ar/htm/site/ ennys/pdf/documento_resultados_2007.pdf

30. Comité Nacional de Medicina del Deporte Infanto-Juvenil, Subcomisión de Epidemiología. Consenso sobre factores de riesgo de enfermedad cardiovascular en pediatría. Sedentarismo. Arch Argent Pediatr 2005;103: 450-63.

31. Tremblay MS, Willms JD. Is the Canadian childhood obesity epidemic related to physical inactivity? Int J Obes Relat Metab Disord 2003;27:1100-5. 